\title{
EchoGéo
}

ECHOGEO Sur le Vif | 2007

\section{Birmanie : vers une démocratisation ou un renforcement de l'appareil dictatorial?}

\author{
Marion Sabrié
}

\section{OpenEdition}

\section{Journals}

\section{Édition électronique}

URL : http://journals.openedition.org/echogeo/2021

DOI : 10.4000/echogeo.2021

ISSN : 1963-1197

\section{Éditeur}

Pôle de recherche pour l'organisation et la diffusion de l'information géographique (CNRS UMR 8586)

\section{Référence électronique}

Marion Sabrié, «Birmanie : vers une démocratisation

ou un renforcement de l'appareil dictatorial ? », EchoGéo [En ligne], Sur le Vif, mis en ligne le 17

décembre 2007, consulté le 20 avril 2019. URL : http://journals.openedition.org/echogeo/2021 ; DOI :

10.4000/echogeo.2021

Ce document a été généré automatiquement le 20 avril 2019.

\section{(2) $\mathbb{Q} \Theta \Theta$}

EchoGéo est mis à disposition selon les termes de la licence Creative Commons Attribution - Pas d'Utilisation Commerciale - Pas de Modification 4.0 International 


\title{
Birmanie : vers une démocratisation ou un renforcement de l'appareil dictatorial?
}

\author{
Marion Sabrié
}

1 La Birmanie est un pays dont la situation politique et économique est rarement évoquée. Les évènements d'août, de septembre et d'octobre 2007 ont, au contraire, conduit à une médiatisation accrue de la question birmane et à des erreurs d'interprétation. Aujourd'hui, « la crise birmane », du fait de la diminution de sa médiatisation, semble terminée. Pourtant, il n'en est rien. La politique de la junte est désormais celle d'une répression discrète. Comment la Junte a-t-elle réagi par rapport à ces évènements? Quelles ont été les réactions internationales, et pourquoi ont-elles été à la fois ambiguës et diverses? Surtout, quelles sont les perspectives géopolitiques pour la Birmanie à court terme? 


\section{L'ASIE DU SUD-EST CONTINENTALE}

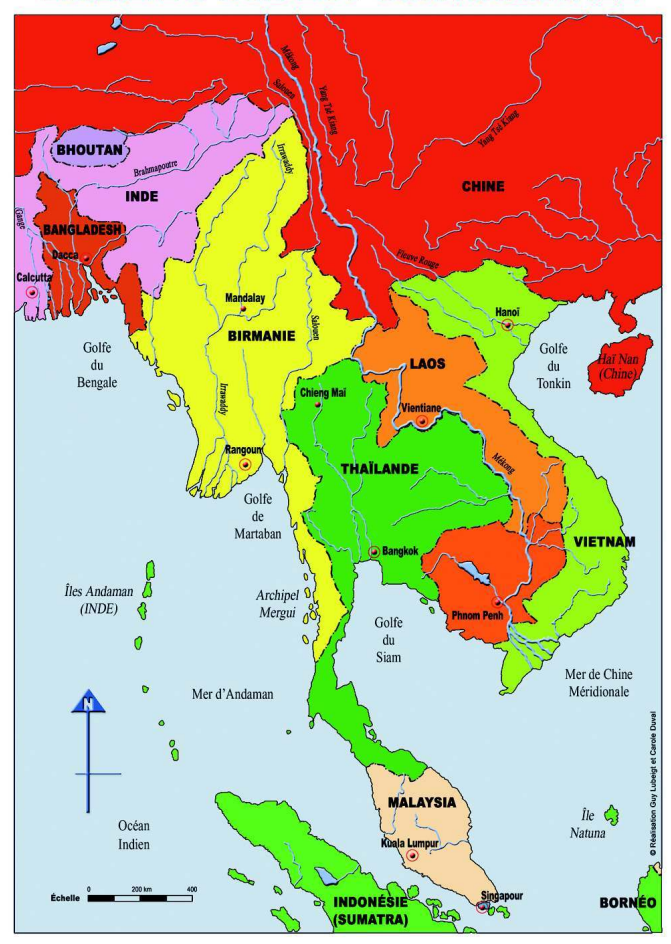

2 Le 15 août 2007, la junte militaire décide d'augmenter le prix du pétrole. Cette hausse a pour conséquence immédiate le doublement des tarifs dans les transports ainsi qu'une hausse de $30 \%$ sur le prix des produits alimentaires de base. L'Occident considère que ce fut l'élément déclencheur des manifestations. Celles-ci, les plus suivies depuis 1988, ne sont pas les premières en Birmanie, mais le phénomène est resté jusqu' alors généralement local et d'ampleur limitée. La nouveauté des mouvements de 2007 réside dans l'implication des moines bouddhistes et la brutale répression. La junte a attendu que les habitants manifestent, pour les filmer et identifier, afin de les arrêter par la suite. Elle a également procédé à l'arrestation de nombreux moines et pillé des monastères. Les moines, dans l'histoire de la Birmanie, ont été aux côtés des Birmans durant la Colonisation britannique, ils ont, de tout temps, par leur engagement politique, incarné la contestation contre le pouvoir central et ils ont été un des fers de lance du nationalisme birman dans un pays bouddhiste à $80 \%$. En septembre 2007, ils ont manifesté car ils vivent de l'aumône quotidienne du peuple. Or, si le peuple se trouve contraint de payer plus cher les produits de base de l'alimentation, il ne pourra plus les nourrir. Les moines ont aussi participé en tant que fils ou frères des manifestants. Du fait de leur participation active aux manifestations, les pagodes et les monastères sont désormais sous étroite surveillance militaire. La pagode principale de Rangoun, Shwedagon Pagoda, endroit très symbolique et emblématique de la Birmanie, a été l'un des lieux majeurs des manifestations. Par « vague safran » il ne faut pas penser l'ensemble des moines birmans (le Sangha) comme une entité unie partageant les mêmes aspirations. Il y a une véritable césure entre les moines influents, hauts placés, et les jeunes novices qui ont été les initiateurs des manifestations. Cette césure existait déjà en 1990 lorsque les novices furent les instigateurs de la "Grève du bol à aumône". A l'inverse, les moines hauts placés sont de connivence avec le gouvernement. C'est d'ailleurs sous le gouvernement du général Ne Win que le Sangha birman fut réorganisé. Depuis 1988, afin d'effacer les souvenirs de la répression, la junte s'efforce de se faire passer pour un gouvernement très 
religieux en offrant des sommes considérables à des monastères, en reconstruisant des édifices religieux, en érigeant des statues et en donnant des célébrations. Désormais, le peuple ne peut plus être dupe, et le Sangha divisé ne peut plus servir de médiateur entre le peuple et la junte militaire.

En raison de la politique répressive de la junte, la question de la démocratie et parallèlement celle des Droits de l'Homme, s'est ajoutée aux problèmes économiques. Les manifestations ont été une opportunité pour plusieurs activistes et membres de l'opposition, notamment de la "Génération 88 ", qui ont initié les évènements de 1988, de revenir sur le devant de la scène et d'instrumentaliser les manifestations à des fins politiques. Certaines manifestations ont aussi été organisées par les moines. Pour atténuer l'impact de ces revendications, des contre-manifestations ont été organisées par la junte afin que les habitants, forcés d'y participer, affichent leur soutien à la paix, la stabilité et l'union nationale.

4 Sur la scène internationale, de nombreux pays ont pris position face à "la crise birmane". Des sanctions préexistaient aux évènements de septembre 2007, comme l'embargo américain mis en place le 22 juillet 1991 pour protester contre la politique de répression du régime birman. En 2003, avaient été votés des restrictions dans les échanges avec la Birmanie, le gel des avoirs financiers birmans dans les banques américaines, l'interdiction de visa aux Etats-Unis pour les membres de la junte, ainsi que l'octroi d'une aide aux militants démocrates de Birmanie. L'Europe a également instauré des sanctions, mais la France joue un rôle ambigu comme l'installation du groupe Total depuis 1995 à Yadana en Birmanie.

5 Néanmoins, les pays voisins de la Birmanie comme l'Inde et surtout la Chine attendent de pouvoir récupérer les exploitations si le groupe Total venait à partir. De plus, des gisements découverts en décembre 2003 dans l'Arakan, constituent une nouvelle ressource qui intéresse fortement les puissances asiatiques. La polémique avait déjà été lancée en mars 2005 lorsque la présence du groupe Total avait été critiquée par des ONG britanniques: la conclusion avait été que son retrait n'affaiblirait en rien la junte militaire et que de nombreuses compagnies asiatiques souhaitaient vivement exploiter les ressources birmanes.

6 Pour l'avenir géopolitique de la Birmanie, plusieurs scenari sont possibles. De la même façon que le nord de la Birmanie est très sinisé, le reste du pays, qui a déjà tendance à être un satellite de la Chine, pourrait passer sous son contrôle. Les Chinois représenteraient 2 $\%$ de la population birmane et jusqu'à $30 \%$ à Mandalay. Mais, malgré l'influence de Beijing, il semble peu probable que les généraux de la junte se laissent manipuler par le gouvernement chinois, du fait de la grande xénophobie des généraux birmans (n'oublions pas les pogroms anti-chinois de 1967 à Rangoun et dans le reste du pays). 


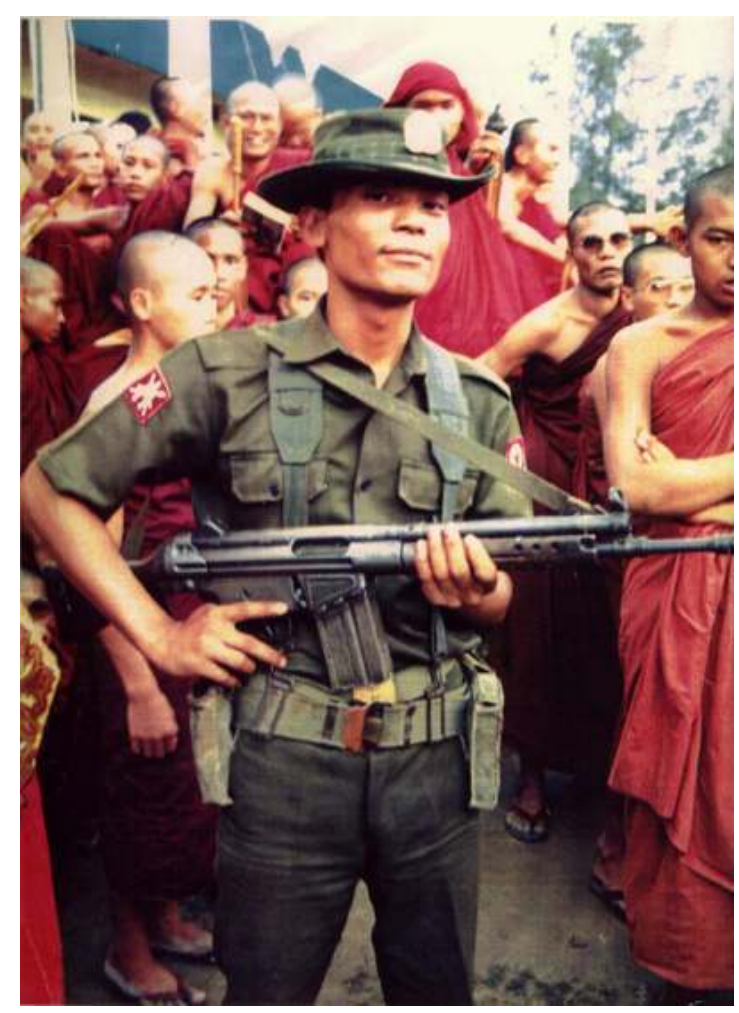

Source : Guy Lubeigt, 1995.

Or, par la répression que la junte a mené contre les manifestants et en particulier contre les moines, très respectés, le pouvoir central dont la réputation à l'intérieur même du pays s'était déjà dégradée, s'est totalement discrédité auprès de son peuple. La volonté de la population de "se débarrasser" de la junte est encore plus grande aujourd'hui qu'en 1988, 19 ans de dictature plus tard. Pourtant, les manifestants sont moins nombreux : cela s'explique par l'affaiblissement du peuple sous les années de dictature, un peuple qui finit par se résigner, et parce que la nouvelle génération née sous la dictature n'a pas connu d'autre réalité.

- Le scénario le plus optimiste serait que les partenaires privilégiés de la Birmanie, comme l'Inde et la Chine, fassent pression sur la junte, et qu'une démocratie y soit instaurée avec Aung San Suu Kyi à sa tête. Or, si les puissances asiatiques ne s'y résolvent pas, c'est que les intérêts qu'elles trouvent à commercer avec la dictature y sont beaucoup plus grands : la stabilité des échanges et de la situation politique. En effet, grâce à la sinisation du nord de la Birmanie et aux infrastructures réalisées par la Chine, les Etats du Yunnan, du Sichuan et Kunming en particulier, ont accès à l'Océan Indien et bénéficient également des revenus de l'exploitation massive de la forêt du nord-est du pays par des entreprises chinoises. L'accès à l'Océan Indien permet à la Chine de conquérir plus facilement le marché potentiel des 200 millions de personnes vivant en Asie du Sud-est continentale, et de profiter des réglementations du marché de l'ASEAN. L'ampleur de la présence chinoise en Birmanie lui vaut le surnom de "futur Etat du Yunnan".

- De ce fait, le scénario qui semble le plus probable est un renforcement de l'appareil dictatorial. Dans cette optique, une nouvelle Constitution, officiellement envisagée le 3 septembre 2003, par le Général Thein Sein ne fera que renforcer le pouvoir militaire. Malgré des défections de militaires qui montrent que la junte n'est plus monolithique et 
qu'il existe bien quelques fissures, toute manifestation est réprimée, et la dictature, établie depuis 1962 et renforcée en 1988, ne semble pas être sur sa fin.

La junte refuse toute discussion malgré les tentatives de l'ONU d'instaurer le dialogue. Le régime birman a en effet renoncé à une proposition onusienne de "rencontre à trois" entre Mr. Gambari, l'envoyé spécial du Secrétaire Général de l'ONU, Aung San Suu Kyi, la présidente de la Ligue Nationale pour la Démocratie et un intermédiaire nommé par la junte, le Général Aung Kyi. Face au refus de la junte, Aung San Suu Kyi a déclaré qu'elle laissait Mr. Gambari se charger des discussions puisque la junte refusait sa présence. La junte lui a demandé d'abandonner son soutien à la politique occidentale des sanctions si elle voulait réellement contribuer à la "réconciliation nationale". Mr. Gambari a terminé ses deux visites en Birmanie qui se sont soldées par un semi-échec. Il a certes à nouveau rencontré Aung San Suu Kyi et le Général Aung Kyi, mais il n’a pas été reçu par le Général Than Shwe qui ne veut pas coopérer et refuse toutes les accusations internationales. Selon la junte, la "crise birmane" (qui n'en est pas une) est une affaire nationale devant se régler à l'intérieur du pays.

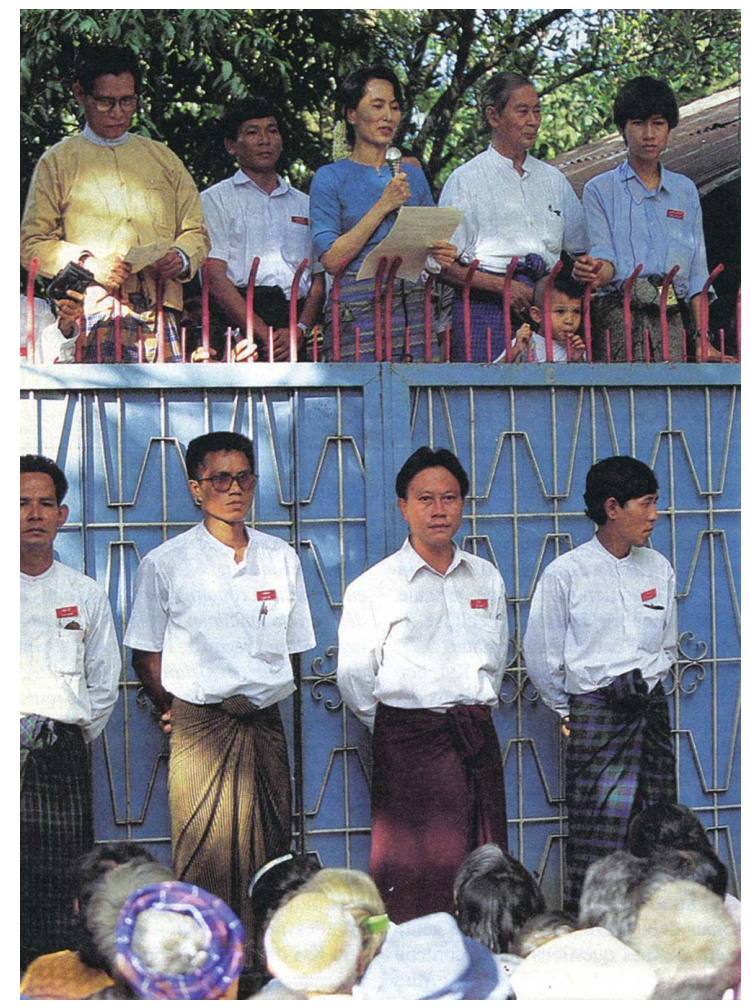

Source : Guy Lubeigt, 2005.

Or, cette situation profite aux deux Grands asiatiques. La Chine, faisant preuve d'une évidente mauvaise foi, partage l'argument de «l'affaire nationale », qui ne se justifie que dans l'optique de la poursuite des échanges économiques. De plus, ayant proclamé son soutien officiel à la junte, elle aurait également engagé des contacts avec des groupes d'opposition en exil : une façon de ne pas perdre ses positions économiques sur place en cas de renversement de la junte militaire.

10 Avec 1647 kilomètres de frontière commune avec la Birmanie, l'Inde a elle aussi de nombreux intérêts. S'y implanter économiquement fait partie de la "New East Policy" indienne qui semble avoir marqué un tournant en 1993 en rompant avec la politique d'abord neutraliste puis isolationniste mise en place durant la dictature 
de Ne Win (de 1962 à 1988). Celle-ci vise surtout à limiter la présence chinoise. L'Inde n'est vraiment intéressée que par le Shwe Gas Project et par la construction d'un gazoduc de l'Arakan à Calcutta, soit en traversant le Bangladesh, soit par les Etats Indiens du Nord-Est où les insurrections dues à des mouvements séparatistes posent problème. L'Inde souhaiterait aussi voir la construction d'une Route transasiatique qui traverserait la Birmanie par un axe Chittagong - Rangoun - Bangkok, projet auquel le Bangladesh tient également. Cela lui permettrait d'accroître ses exportations et ses importations. La question des échanges frontaliers demeure un problème récurrent. De nombreuses visites bilatérales se sont soldées par un échec aussi bien concernant la question des migrations et des réfugiés que celle des échanges commerciaux.

11 Francis Christophe résume bien la situation de la Birmanie : on a assisté au "passage d'une narcodictature à une gazodictature". L'attitude de la communauté internationale apparaît très équivoque par rapport au secteur gazier. Mais c'est un cercle vicieux : si une entreprise laisse sa place, une autre la prendra. La Birmanie est prise dans un étau entre l'Inde et la Chine principalement, et entre ses autres partenaires économiques voisins dont chacun veut sa part du gâteau. Et la Chine a déjà une longueur d'avance sur l'Indequi avait pris parti pour les manifestants en 1988 et qui s'exprime aujourd'hui d'une toute autre façon par la voix de son chef de la diplomatie: "en tant que voisin proche et amical, l'Inde espère voir une Birmanie en paix, stable et prospère", insistant ainsi sur les préoccupations économiques de son pays. Les deux grands ne sont pas prêts à faire des concessions.

Renaud Egreteau, dans un article de Libération daté du 16 octobre 2007 et intitulé " Les sanctions ne peuvent pas fonctionner", développe une idée prenant le contrepied denotre façon d'appréhender l'avenir birman: "Face à un régime isolationniste et paranoïaque, c'est peut-être l'inverse du boycott qu'il faut tenter : quelle serait la réaction des généraux birmans, si la communauté internationale se décidait à investir massivement dans l'économie, le social, la culture, l'éducation et l'humanitaire ?». Peut-être a-t-il paradoxalement raison...

Les défections au sein de l'armée représentent une menace beaucoup plus grande pour la junte militaire que les manifestations populaires et que les sanctions ou autres "intimidations" internationales. De plus, la Birmanie représente peu d'enjeux pour de nombreux pays qui en appellent aux sanctions. Au contraire, elle est très importante pour les gouvernements qui ne se prononcent pas en faveur des sanctions, vivement intéressés par la position carrefour de la Birmanie à la charnière des mondes indien, chinois et sud-est asiatique et par ses nombreuses ressources. Le principal problème qui se pose aux occidentaux, c'est que les sanctions, qui voudraient pénaliser la junte militaire, touchent d'abord directement le quotidien du peuple birman, déjà durement éprouvé par la dictature.

Des manifestations anti-gouvernements paraissaient très peu probables avant les évènements de l'automne 2007, même si les prémices de la révolte étaient perceptibles. L'auteure de ces lignes avait conclu son travail de recherches sur l'Arakan en juin 2007 par ces mots: “mais la révolte n'a pas encore toutes ses chances car l'armée et le gouvernement central sont là pour contrer toute opposition." La question de l'unité nationale demeure, car si la junte était vaincue, 
de la même façon que l'histoire birmane montre un fédéralisme avorté, sans pouvoir central fort, la Birmanie risquerait d'être un pays éclaté en plusieurs Etats. N'oublions pas que $30 \%$ de le population sont des minorités (69\% sont Birmans - en comptabilisant aussi les Arakanais, 9 \% sont Shan, 7 \% Kayin, 4 \% Kayah, 3 \% Chin, 3 \% Kachin et 3 \%Môn). Mais pour le moment, il semblerait que rien ne change en Birmanie malgré l'optimisme avec lequel certains voient les retrouvailles entre Aung San Suu Kyi et les membres de son parti. Un quotidien titrait justement: "Opération Séduction" car la junte qui n'est pas aussi isolée qu'en 1988, fait semblant de jeter du lest à la communauté internationale. De la même façon, les évènements de 1988 n'avaient été qu'un coup d'épée dans l'eau et n'avaient mené qu'à l'exil ou à l'arrestation des opposants au pouvoir et les élections de 1990 n'avaient pas été suivies par la formation d'un nouveau gouvernement. Sur la voie de la démocratisation, la route semble encore bien longue pour une société aussi militarisée que la Birmanie.

\section{RÉSUMÉS}

Au centre des intérêts économiques et stratégiques sino-indiens, les pressions occidentales apparaissent inefficaces contre la junte militaire birmane. Malgré les efforts de dialogue de l'ONU, l'appareil dictatorial semble se renforcer. En partie par la faute d'une scène internationale divisée, la route semble encore longue sur la voie de la démocratisation pour une société aussi militarisée que la Birmanie.

In the center of the Sino-Indian economical and strategical interests, western sanctions against the Burmese military junta appear ineffective. While the ONU makeefforts to establish a dialogue, the dictatorship seems to reinforce itself. Partly because of the divisions in the international scene, the road to democratization remains far away for the Burmesemilitary society.

\section{INDEX}

Mots-clés : Birmanie, democratisation, dictature, moines, répression

Keywords : Burma, democratization, dictaorship, monks, repression

\section{AUTEUR}

\section{MARION SABRIÉ}

Marion Sabrié (marion.sabrie@no-log.org) est en thèse à l'Université Paris Sorbonne sous la direction d'Olivier Sevin. Son terrain est la Birmanie dont elle maîtrise la langue 